\title{
Using molecular descriptors for assisted screening of heterologous competitive antigens to improve the sensitivity of ELISA for detection of enrofloxacin in raw milk
}

\author{
Song Hu, ${ }^{1}$ Bolong Fang, ${ }^{1}$ Zhen Huang, ${ }^{1}$ Yuan Chen, ${ }^{1}$ Daofeng Liu, ${ }^{2}$ Keyu Xing, ${ }^{1}$ Juan Peng, ${ }^{3 *}$ \\ and Weihua Lai ${ }^{1 *}$ \\ ${ }^{1}$ State Key Laboratory of Food Science and Technology, Nanchang University, Nanchang 330047, China \\ ${ }^{2}$ Jiangxi Province Center for Disease Control and Prevention, Nanchang 330047, China \\ ${ }^{3}$ School of Food Science, Nanchang University, Nanchang 330047, China
}

\section{ABSTRACT}

The use of the heterologous competitive strategy has become a vital method to improve the sensitivity of ELISA. In this work, we prepared an anti-enrofloxacin (ENR) mAb with ENR-bovine serum albumin (BSA) as immunogen. The molecular descriptors of quinolones were then used to screen heterologous coating antigens for the detection of ENR based on an ensemble learning method to improve the sensitivity of the ELISA. Results indicated that 6 of the 7 selected heterologous competitive antigens could enhance the sensitivity of ELISA. The ELISA sensitivity for the detection of ENR with sarafloxacin-BSA as heterologous coating antigen was improved 10-fold (in PBS) and 6-fold (in milk) compared with that with ENR-BSA as homologous antigen. The strategy can effectively screen suitable heterologous competitive antigens to improve the sensitivity of ELISA, followed by preparation of $\mathrm{mAb}$ with no additional modification to the corresponding immunogen.

Key words: sensitivity of ELISA, heterologous competitive antigen, molecular descriptors, enrofloxacin

\section{INTRODUCTION}

In recent years, rapid detection for target identification based on antigen-antibody (Ag-Ab) recognition has developed significantly in the fields of point-of-care testing (Huang et al., 2017), environmental monitoring (Tang et al., 2017), and food safety testing (Yu et al., 2018). Compared with instrumental analysis methods,

Received November 26, 2018.

Accepted February 10, 2019.

*Corresponding authors: pengjuan2016@163.com and talktolaiwh@ 163.com such as HPLC (Turiel et al., 2006) and chromatography coupled with mass spectrometry (Bianchi et al., 2007; Guidi et al., 2018), rapid detection does not require expensive instruments, skilled personnel, or considerable detection time (Hu et al., 2017). Enzyme-linked immunosorbent assay is the method commonly used to screen targets in a rapid detection field due to its rapidity and convenience. However, its sensitivity could not meet the maximum residue level (Colgrave et al., 2014). Hence, improving the sensitivity of ELISA is important and necessary.

Preparing a mAb with high affinity to the corresponding antigen is a common approach to enhance ELISA sensitivity but requires tedious work. The heterologous competitive strategy, wherein a heterologous competitive antigen is used as the coating antigen, might enhance the sensitivity of ELISA because the affinity of $\mathrm{mAb}$ to a heterologous competitive antigen is less than the affinity to a homologous antigen (Hosoda et al., 1981; Goodrow et al., 1990; Rao and Taraporewala, 1992; Rassaie et al., 1992; Zhang et al., 2007). Most researchers have used heterologous coating antigens based on numerous experiments; however, this strategy requires considerable time and money. Hence, developing a simple and reliable strategy to select heterologous coating antigen is urgently needed to improve the sensitivity of ELISA.

Monoclonal antibodies recognize heterologous haptens based on their different structures (Wang et al., 2007; Chen et al., 2017a). Molecular descriptors are the result of a logical and mathematical operation that converts chemical information encoded within a symbolic representation of a molecule into a useful numerical value (Bober et al., 2011). The structural information of a molecule can be represented by molecular descriptors based on several parameters, including physical properties, atom count, and bond counts, which can be used to distinguish different molecules. 
Enrofloxacin (ENR), a common quinolone, is widely used for disease prevention in livestock (Barberio et al., 2016; Yang et al., 2016; Ershadi et al., 2017; Peng et al., 2017). However, the presence of ENR residues in milk arouses public health concerns because of its negative effect on the human intestine and the risk of antibiotic resistance (Zhao et al., 2008; Pereira et al., 2014; Otero et al., 2016; Ershadi et al., 2017). Therefore, the European Union (EMEA, 2002), World Health Organization (WHO), Japan (Ministry of Health, Labor and Welfare), and China (Ministry of Agriculture) have set maximum residue limits (MRL) for ENR in milk, to decrease the risk to public health associated with consumption of ENR residues, at 100, 40, 20, and $100 \mu \mathrm{g} / \mathrm{L}$, respectively. These low values require the development of ELISA that are sensitive enough to monitor and detect ENR in milk sample. Moreover, there are structural analogs of ENR, which allow us to explore the relationship between heterologous competitive strategy and ELISA sensitivity.

In this work, we first prepared an anti-ENR mAb. Then, we used the ensemble learning method to explore the relationship between heterologous competitive strategy and ELISA sensitivity by introducing molecular descriptors for quinolones. This study provides practical guidance for selecting suitable heterologous coating antigens to improve the sensitivity of other immunoassays.

\section{MATERIALS AND METHODS}

\section{Materials and Reagents}

Balofloxacin (BAL), besifloxacin (BES), cinoxacin (CIN), clinafloxacin (CLI), danofloxacin (DAN), enrofloxacin (ENR), fleroxacin (FLE), gemifloxacin (GEM), lomefloxacin (LOM), marbofloxacin (MAR), moxifloxacin (MOX), nalidixic acid (NAL), norfloxacin (NOR), orbifloxacin (ORB), oxolinic acid (OXO), pefloxacin (PEF), prulifloxacin (PRU), pipemidic acid (PIP), pazufloxacin (PAZ), sarafloxacin (SAR), sitafloxacin (SIT), sparfloxacin (SPA), florfenicol (FLO), sulfamethazine (SMZ), and tetracycline (TET) were obtained from J\&K Scientific Ltd. (Shanghai, China). The structure of these drugs is given in Supplemental Figure S1 (https://doi.org/ 10.3168/jds.2018-16048). Bovine serum albumin, ovalbumin (OVA), 1-(3-dimethylaminopropyl)-3-ethylcarbodiimide, $N$-hydroxysuccinimide, dimethylformamide, Freund's complete and incomplete adjuvants, 3,3',5,5'-tetramethylbenzidine (TMB), Tween-20, and gelatin were purchased from Sigma-Aldrich (St. Louis, MO). Horseradish peroxidase-labeled goat anti-mouse
IgG was obtained from Beijing Zhongshan Biotechnology Inc. (Beijing, China). Hypoxanthine-aminopterinthymidine and hypoxanthine-thymidine supplements and other cell culture reagents were purchased from Life Technologies Corporation (Shanghai, China). The raw milk was free of ENR, as validated by HPLC-MS/ MS, was provided by Jiangxi Sunshine Dairy Co. Ltd. (Jiangxi, China). Twenty-five raw milk samples for the validation experiment were collected from Nanchang (Jiangxi Sunshine Dairy Co. Ltd.). Other reagents and chemicals were obtained from the National Pharmaceutical Group Chemical Reagent Co. Ltd. (Shanghai, China).

\section{Instrumentation and Supplies}

The ELISA was performed in polystyrene 96-well microtiter plates (Bio Basic Inc., Markham, ON, Canada). A microplate reader (Thermo LabSystems, Beverly, MA) was used to measure the optical density (OD). The UV-visible (UV-vis) spectra of antigens were obtained with a UV-vis spectrometer (UV-2300, Shimadzu, Kyoto, Japan).

\section{Buffer}

Phosphate-buffered saline $(0.01 M, \mathrm{pH}$ 7.4) consisted

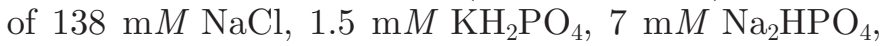
and $2.7 \mathrm{mM} \mathrm{KCl}$. The wash buffer was PBS with $0.05 \%$ Tween 20. As a coating buffer, $0.05 M$ carbonate buffer (CB, $15 \mathrm{mM} \mathrm{Na} \mathrm{NaO}_{3}$ and $35 \mathrm{mM} \mathrm{NaHCO}$, pH 9.6) was used. The blocking buffer was $\mathrm{CB}$ with $0.2 \%$ of gelatin and $0.05 \%$ (vol/vol) Tween-20. The antibody dilution solution was $0.01 \mathrm{M}$ PBS containing $0.05 \%$ (vol/vol) with Tween-20 and $0.1 \%$ gelatin (wt/vol). The stopping solution was $2 M \mathrm{H}_{2} \mathrm{SO}_{4}$.

\section{Synthesis of Immunogen and Coating Antigens}

An immunogen was prepared by conjugating ENR with BSA (Tochi et al., 2015). Briefly, a mixture consisting of $1 \mathrm{~m} M$ ENR, $5 \mathrm{~m} M$ 1-(3-dimethylaminopropyl)3 -ethyl-carbodiimide, and $5 \mathrm{mM} \mathrm{N}$-hydroxysuccinimide was dissolved in $200 \mu \mathrm{L}$ of dimethylformamide and stirred for $8 \mathrm{~h}$ at room temperature. The mixture was added dropwise into $2 \mathrm{~mL}$ of $0.01 M \mathrm{CB}$ solution with $3.35 \mathrm{mg} / \mathrm{mL}$ BSA. After stirring overnight, the reactants were dialyzed for $3 \mathrm{~d}$ in $0.01 \mathrm{M}$ PBS to obtain the immunogen (ENR-BSA). Meanwhile, the coating antigen (ENR-OVA) used in the subsequent sera and cell screening was synthesized with OVA as ligand protein by using the method described above. 


\section{Preparation of $m A b$}

The protocol for carrying out animal experiments was approved by Nanchang University Animal Ethics Committee and all the ethical requirements to conduct the experiment were met. All procedures were conducted in compliance with protocols provided and approved by the Animal Care Review Committee (approval number 0064257) of Nanchang University (Jiangxi, China).

Five female BALB/c mice obtained from the Shanghai Laboratory Animal Center (Shanghai, China) were immunized through continuous multipoint subcutaneous injection with immunogen (Peng et al., 2016). The mice were first immunized using $100 \mu \mathrm{g}$ of immunogen emulsified in Freund's adjuvant. After 1 mo, 5 booster immunizations were administered, 1 every $21 \mathrm{~d}$ with $50 \mu \mathrm{g}$ of immunogen emulsified in incomplete Freund's adjuvant for each mouse. Seven days after the last immunization, the mice were tail bled, and their sera were evaluated through indirect competitive ELISA (icELISA) with ENR-OVA as the coating antigen. The mouse that exhibited good affinity to ENR-OVA and excellent inhibition of ENR received a final intraperitoneal injection of $25 \mu \mathrm{g}$ of immunogen. Three days later, the mouse was killed by cervical dislocation and cell fusion procedures were performed. Briefly, the spleen was removed, and the splenocytes were isolated and fused with Sp2/0 myeloma cells. After 7 $\mathrm{d}$ of culture in hypoxanthine-aminopterin-thymidine and hypoxanthine-thymidine solutions, supernatants of the hybridoma cells were screened by icELISA; the selected hybridoma cells were cloned 3 times via limiting dilution before ascites production. The ascites fluid was purified with caprylic acid and ammonium sulfate precipitation (Perosa et al., 1990) with minor modifications. Briefly, $1 \mathrm{~mL}$ of ascites fluid was diluted with 2 $\mathrm{mL}$ of $0.06 M$ acetate buffer and the $\mathrm{pH}$ adjusted to 4.5 with $1 \mathrm{M} \mathrm{HCl}$. Thirty-three microliters of caprylic acid was then added dropwise with vigorous mixing. After stirring at room temperature for $1.5 \mathrm{~h}$, the mixtures were centrifuged at $14,800 \times g$ at $4^{\circ} \mathrm{C}$ for $20 \mathrm{~min}$. The supernatant was then collected and the $\mathrm{pH}$ adjusted to 7.4 with $1 \mathrm{M} \mathrm{NaOH}$. The solution was stirred at $4^{\circ} \mathrm{C}$ for $30 \mathrm{~min}$ and ammonium sulfate $(0.277 \mathrm{~g} / \mathrm{mL})$ was added. The precipitated antibodies collected by centrifugation $\left(10,300 \times g\right.$ at $4^{\circ} \mathrm{C}$ for $\left.1 \mathrm{~h}\right)$ were then resuspended in $1 \mathrm{~mL}$ of $\mathrm{PBS}$ ( $\mathrm{pH}$ 7.4). The prepared antibodies were stored at $4^{\circ} \mathrm{C}$ for further experiments.

\section{ELISA Procedure}

An icELISA was performed to evaluate the affinity of the antibodies to the analyte in serum or superna- tants of hybridoma cells as described previously (Peng et al., 2015). The icELISA procedures were as follows: $100 \mu \mathrm{L} /$ well of ENR-OVA solution $(0.1 \mu \mathrm{g} / \mathrm{mL}$, diluted in $0.01 M \mathrm{CB}$ ) was added to the 96 -well plates and incubated at $37^{\circ} \mathrm{C}$ for $2 \mathrm{~h}$. The plates were washed 3 times with $0.01 M$ PBS-Tween and blocked with 100 $\mu \mathrm{L} /$ well of $0.01 M \mathrm{CB}$ containing $0.2 \%$ (wt/vol) gelatin at $37^{\circ} \mathrm{C}$ for $2 \mathrm{~h}$. After washing, $50 \mu \mathrm{L}$ of ENR (0, 0.05, $0.1,0.5,1,5$, and $10 \mathrm{ng} / \mathrm{mL}$ ) in PBS and $50 \mu \mathrm{L}$ of 0.1 $\mu \mathrm{g} / \mathrm{mL} \mathrm{mAb}$ in antibody dilution solution were added and incubated at $37^{\circ} \mathrm{C}$ for $30 \mathrm{~min}$. The plates were then washed 3 times, and $100 \mu \mathrm{L} /$ well of horseradish peroxidase-conjugated goat anti-mouse $\operatorname{IgG}$ (diluted to 1:3,000 with antibody dilution buffer) was added. After incubation at $37^{\circ} \mathrm{C}$ for $30 \mathrm{~min}$, the plates were washed 4 times, and then $100 \mu \mathrm{L} /$ well of TMB solution $(2 \mathrm{~mL}$ of $0.06 \%$, wt/vol, TMB in glycol with $10 \mathrm{~mL}$ of $0.1 \mathrm{M}$ citrate phosphate buffer, $\mathrm{pH} 5.0$, containing $1.8 \mu \mathrm{L}$ of $30 \%$ hydrogen peroxide) was added. Finally, $50 \mu \mathrm{L}$ of 2 $M \mathrm{H}_{2} \mathrm{SO}_{4}$ was added to stop the reaction after incubation at $37^{\circ} \mathrm{C}$ for $15 \mathrm{~min}$. The OD was determined at 450 $\mathrm{nm}$ by using a microplate reader.

\section{Evaluation of the Sensitivity and Cross-Reactivity of the Training Samples}

The icELISA was used to evaluate the sensitivity of ELISA for the detection of 18 quinolones (training samples) and their corresponding cross-reactivities (Chen et al., 2017b). The concentration of the standard that provided a tracer binding inhibition in $\mathrm{OD}$ values at $50 \%$ was defined as the $\mathrm{IC}_{50}$ value; thus, ELISA sensitivity is represented by the $\mathrm{IC}_{50}$ value. The crossreactivity $(\mathbf{C R})$ values were calculated as follows: $\mathrm{CR} \%$ $=\left(\mathrm{IC}_{50}\right.$ of analyte $/ \mathrm{IC}_{50}$ of analog $) \times 100$. Training samples, including ENR, PEF, PIP, NOR, DAN, FLE, BAL, BES, CIN, CLI, GEM, LOM, MAR, MOX, NAL, ORB, OXO, and PAZ, were tested to determine their corresponding $\mathrm{CR}$. The $\mathrm{IC}_{50}$ values were calculated using OriginPro 2016 (OriginLab Corp., Northampton, MA).

\section{Machine Learning}

Calculation of Molecular Descriptors of the Training Samples. Molecular descriptors of the training samples were calculated using the Molecule Operating Environment (MOE) 2016.10 package (Chemical Computing Group Inc., Montreal, QC, Canada) including (a) notation and terminology, (b) physical properties, (c) Hückel theory descriptors, (d) subdivided surface areas, (e) atom counts and bond counts, (f) Kier and Hall connectivity and kappa shape 
indices, (g) adjacency and distance matrix descriptors, (h) pharmacophore feature descriptors, and (i) partial charge descriptors.

PCA for Molecular Descriptors. Principal components analysis (PCA) is a common machine learning method used in unsupervised exploratory data analysis. Principal components, which are obtained through PCA of high-dimension data, can search for internal relationships among the characteristics of a set of objects, enabling a drastic reduction in dimensions of the original raw data (Ballabio, 2015). The PCA for molecular descriptors was run on MATLAB 2015a (The MathWorks Inc., Natick MA).

Classification Learning. The corresponding principal components of 18 quinolones were used to train the machine with the ensemble learning method to acquire known molecular descriptor-CR interaction (0 represents $\mathrm{CR}<0.01$, whereas 1 represents $\mathrm{CR}>0.07$ ). A CR $<0.01$ means the drugs cannot be recognized, whereas $\mathrm{CR}>0.07$ indicates the corresponding quinolones can be captured by mAb in the ELISA system. Operations involving ensemble learning were performed using the corresponding embedded procedure in MATLAB 2015a (The MathWorks Inc.).

\section{Prediction and Verification of $C R$ for Testing Samples}

The molecular descriptors of the testing samples, including 4 quinolones (PRU, SAR, SIT, and SPA) and 3 other antibiotics (FLO, SMZ, and TET), were calculated using the method described in the Calculation of Molecular Descriptors of the Training Samples section. After PCA of the molecular descriptors of the testing samples, the ensemble learning method was used to obtain unknown molecular descriptor-CR interactions based on the known one. Subsequently, the CR of the testing samples were verified by icELISA. The icELISA procedures and CR calculations were similar to those described in Evaluation of the Sensitivity and CrossReactivity of the Training Samples.

\section{Sensitivity of ELISA to Heterologous Competitive Antigen of Testing and Training Samples}

The coating antigens of 8 quinolones categorized as "1" in the testing and training samples were prepared as described in the Synthesis of Immunogen and Coating Antigens section; the antigens included homologous coating antigens (ENR-BSA) and heterologous competitive antigens (DAN-BSA, PRU-BSA, NOR-BSA, PEF-BSA, FLE-BSA, SAR-BSA, and PIP-BSA). The
$\mathrm{IC}_{50}$ values of ELISA for detection of ENR were determined using these 8 antigens as coating antigens.

\section{Analysis of Recognition Capability Between mAb and Heterologous Competitive Antigens}

The docking poses of quinolones and lysine were used for molecular mechanics minimization by using the MOE 2016.10 package to elucidate the recognition capability between $\mathrm{mAb}$ and heterologous coating antigens of interest. The force field in the molecular mechanics minimization was MMFF94x with an 8-Å cutoff for non-bonded interactions. The conformations after the preliminary minimization were further determined to achieve a more accurate geometric optimization and frequency analysis at the $\mathrm{HF} / 6-31 \mathrm{G}(\mathrm{d})$ level by using the Gaussian 09 package (Gaussian Inc., Wallingford, $\mathrm{CT}$ ), ultimately obtaining the minimum energy conformations of all condensation products. Moreover, the atomic point charges and electrostatic potential were calculated using the Gaussian 09 package at the same level and viewed using the GaussView 5.0 package (SemiChem Inc., Wallingford, CT).

\section{Detection of ENR in Raw Milk Using Suitable Heterologous Competitive Antigens}

Enrofloxacin was added into ENR-negative raw milk at $0.05,0.10,0.25,0.50,1.00,2.50,5.00,10.00,25.00$, 50.00 , and $100.00 \mathrm{ng} / \mathrm{mL}$. The sensitivity of ELISA for detection of ENR in negative and positive samples was based on ENR-BSA and 6 suitable heterologous competitive antigens as coating antigens, respectively (Wang et al., 2014). The corresponding $\mathrm{IC}_{50}$ was calculated using OriginPro 2016.

Then, ENR (0.1, 0.5, and $2 \mathrm{ng} / \mathrm{mL})$ was added to raw milk. The recovery and coefficient of variation $(\mathrm{CV})$ of ENR in raw milk were quantitatively analyzed on the ELISA system. Finally, 25 unspiked milk samples were selected and determined by the proposed ELISA and HPLC-MS/MS to verify the reliability of the method.

\section{RESULTS AND DISCUSSION}

\section{UV-Vis Spectra of Antigens}

The UV-vis spectra of ENR-BSA and ENR-OVA are shown in Supplemental Figure S2 (https://doi.org/10 $.3168 / \mathrm{jds} .2018-16048$ ). The spectra indicated that the antigens display the characteristic absorption peaks of both the haptens and ligand proteins, demonstrating the successful preparation of the antigens (Tochi et al., 2015). 
Table 1. Median inhibitory concentration $\left(\mathrm{IC}_{50}\right)$ of ELISA and the corresponding cross-reactivity values with ENR-BSA as coating antigen for the detection of 18 quinolones (haptens)

\begin{tabular}{lcc}
\hline Hapten $^{1}$ & $\mathrm{IC}_{50}(\mathrm{ng} / \mathrm{mL})$ & Cross-reactivity $(\%)$ \\
\hline ENR & 1.32 & 100 \\
PEF & 32.46 & 4.05 \\
PIP & 69.59 & 1.89 \\
NOR & 352.87 & 0.37 \\
DAN & 980.46 & 0.13 \\
FLE & $1,646.67$ & 0.07 \\
BAL & $>10,000$ & $<0.01$ \\
BES & $>10,000$ & $<0.01$ \\
CIN & $>10,000$ & $<0.01$ \\
CLI & $>10,000$ & $<0.01$ \\
GEM & $>10,000$ & $<0.01$ \\
LOM & $>10,000$ & $<0.01$ \\
MAR & $>10,000$ & $<0.01$ \\
MOX & $>10,000$ & $<0.01$ \\
NAL & $>10,000$ & $<0.01$ \\
ORB & $>10,000$ & $<0.01$ \\
OXO & $>10,000$ & $<0.01$ \\
PAZ & $>10,000$ & $<0.01$ \\
\hline
\end{tabular}

${ }^{1} \mathrm{ENR}=$ enrofloxacin; $\mathrm{PEF}$ = pefloxacin; $\mathrm{PIP}=$ pipemidic acid; $\mathrm{NOR}$ $=$ norfloxacin $; \mathrm{DAN}=$ danofloxacin $; \mathrm{FLE}=$ fleroxacin $; \mathrm{BAL}=$ balofloxacin; $\mathrm{BES}=$ besifloxacin; CIN = cinoxacin; $\mathrm{CLI}=$ clinafloxacin; GEM = gemifloxacin; $\mathrm{LOM}=$ lomefloxacin; $\mathrm{MAR}=$ marbofloxacin; $\mathrm{MOX}=$ moxifloxacin $; \mathrm{NAL}=$ nalidixic acid $; \mathrm{ORB}=$ orbifloxacin; $\mathrm{OXO}=$ oxolinic acid PAZ $=$ pazufloxacin .

\section{Evaluation of $m A b$ Performance}

Data obtained during limiting dilution for cell screening are shown in Supplemental Tables S1 and S2 (https: //doi.org/10.3168/jds.2018-16048). A sigmoid curve for ENR detection (Supplemental Figure S3; https://doi .org/10.3168/jds.2018-16048) was obtained with ENROVA as the coating antigen. The performance of the $\mathrm{mAb}$ (represented by $\mathrm{IC}_{50}$ of ELISA) in detecting 18 quinolones was determined by icELISA with ENR-BSA as coating antigen and calculated using the OriginPro 2016 software (Table 1 ). The $\mathrm{IC}_{50}$ values for ENR, PEF, PIP, NOR, DAN, and FLE of ELISA were 1.32, 32.46, $69.59,352.87,980.46$, and $1,646.67 \mathrm{ng} / \mathrm{mL}$, respectively, and their corresponding CR were $>0.07 \%$. However, the $\mathrm{IC}_{50}$ values for the detection of BAL, BES, CIN, CLI, GEM, LOM, MAR, MOX, NAL, ORB, OXO, and PAZ were $>10,000 \mathrm{ng} / \mathrm{mL}(\mathrm{CR}<0.01 \%)$, indicating that these antigens could not be recognized by the $\mathrm{mAb}$ on the ELISA system.

\section{Machine Learning for Training Samples Based on Molecular Descriptors}

The recognition abilities of $\mathrm{mAb}$ varied for each training sample because of structural differences among quinolones (Wang et al., 2007). Molecular descriptors could be used to distinguish analogs. In this work, we first used PCA to reduce the dimension of molecular descriptors to accurately search for the internal relationship between CR and molecular descriptors (Luo et al., 2017). Subsequently, the CR of testing samples were predicted, and the internal relationship between $\mathrm{CR}$ and $10 \mathrm{PC}$ of molecular descriptors for the training samples was determined. In Supplemental Table S3 (https://doi.org/10.3168/jds.2018-16048), the CR based on the machine learning results for PRU, SAR, SIT, SPA, FLO, SMZ, and TET were 1, 1, 0, 0, 0, 0, and 0 , respectively.

We then assessed the prediction performance of the ensemble learning method in terms of accuracy. Accuracy of the ensemble learning method is defined here as the number of correct classifications $\times 100 \% /$ total number of testing samples. The experimental classifications of testing samples were verified using icELISA. In Table 2, the CR of PRU and SAR were 2.86 and 1.44, respectively, whereas those of SIT, SPA, FLO, SMZ, and TET were $<0.01$, indicating that the icELISA classifications of PRU, SAR, SIT, SPA, FLO, SMZ, and TET were $1,1,0,0,0,0$, and 0 , respectively. These results illustrated that the ensemble learning method is highly capable of performing our prediction task (accuracy $=100 \%$, Figure 1 ).

We compared the ensemble learning method with 4 other state-of-the-art methods commonly used in classification prediction tasks (Supplemental Figure S4; https://doi.org/10.3168/jds.2018-16048), including deep linear discriminant analysis (DLDA; Stuhlsatz et al., 2012), K nearest neighbors algorithm (KNN; Shen and Chou, 2005), support vector machine (SVM; Ukil, 2002), and naive Bayes classifiers (NBC; Rosen et al., 2011). Our results showed that ensemble learning consistently outperformed the other methods, with $29 \%$ higher accuracy than DLDA and NBC and $14 \%$ higher accuracy than KNN and SVM. These data also illustrated that ensemble learning could be a reliable classification approach for our prediction task.

Table 2. Median inhibitory concentration $\left(\mathrm{IC}_{50}\right)$ of ELISA and crossreactivity values with ENR-BSA as coating antigen for the detection of the testing samples

\begin{tabular}{lcc}
\hline Hapten $^{1}$ & $\mathrm{IC}_{50}(\mathrm{ng} / \mathrm{mL})$ & Cross-reactivity $(\%)$ \\
\hline ENR & 1.32 & 100 \\
PRU & 46.09 & 2.86 \\
SAR & 90.99 & 1.44 \\
SIT & $>10,000$ & $<0.01$ \\
SPA & $>10,000$ & $<0.01$ \\
FLO & $>10,000$ & $<0.01$ \\
SMZ & $>10,000$ & $<0.01$ \\
TET & $>10,000$ & $<0.01$
\end{tabular}

${ }^{1} \mathrm{ENR}=$ enrofloxacin; PRU $=$ prulifloxacin; $\mathrm{SAR}=$ sarafloxacin; SIT $=$ sitafloxacin; SPA = sparfloxacin; $\mathrm{FLO}=$ florfenicol; $\mathrm{SMZ}=$ sulfamethazine; TET $=$ tetracycline. 


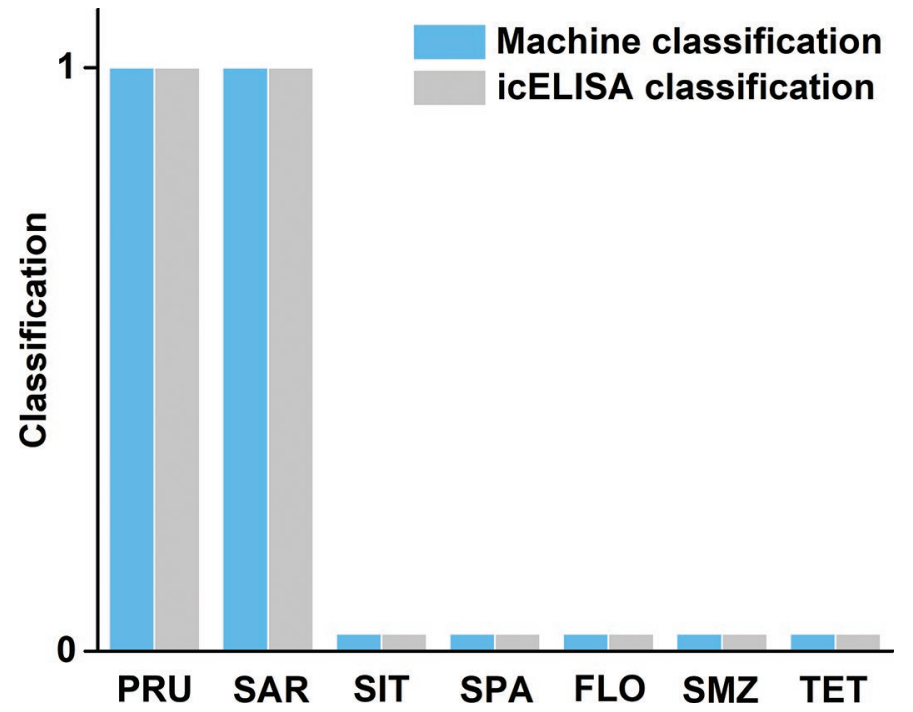

Figure 1. The performance of machine classification based on ensemble learning method compared with the performance of indirect competitive (ic)ELISA classification. 0 represents cross-reactivity $<0.01$, and 1 represents cross-reactivity $>0.07$. PRU $=$ prulifloxacin $\mathrm{SAR}=$ sarafloxacin $; \mathrm{SIT}=$ sitafloxacin $; \mathrm{SPA}=$ sparfloxacin $; \mathrm{FLO}=$ florfenicol; SMZ $=$ sulfamethazine; TET $=$ tetracycline

\section{Sensitivity of ELISA in the Presence of Heterologous Coating Antigens}

We speculated that the heterologous coating antigens with quinolone drugs (category 1 from training samples and testing samples) as haptens could become heterologous coating antigens. Hence, we first prepared 7 heterologous coating antigens (the UV-vis spectra of the heterologous coating antigens are shown in Supplemental Figure S5; https://doi.org/10.3168/ jds.2018-16048). We subsequently evaluated the $\mathrm{IC}_{50}$ values of ELISA with the use of these 7 heterologous coating antigens. As shown in Table 3 , the $\mathrm{IC}_{50}$ values of ENR-BSA, SAR-BSA, FLE-BSA, PEF-BSA, NORBSA, PRU-BSA, and DAN-BSA values of ELISA (i.e., as coating antigens, respectively) for ENR detection were $1.32,0.13,0.21,0.26,0.36,0.49$, and $1.28 \mathrm{ng} / \mathrm{mL}$, respectively.

These results indicated that the sensitivity of ELISA can be improved by using the above 6 heterologous coating antigens of interest, which were categorized as "1" in the training and testing samples based on machine learning results. Table 3 shows that the $\mathrm{IC}_{50}$ of ELISA when SAR-BSA was used as heterologous coating antigen was $0.13 \mathrm{ng} / \mathrm{mL}$, whereas that when ENR-BSA was used as coating antigen was $1.32 \mathrm{ng} /$ $\mathrm{mL}$. Moreover, the corresponding coating antigens when the 17 quinolones of category 0 were used as haptens could not be recognized by $\mathrm{mAb}$ on the ELISA system (Supplemental Figure S6; https://doi.org/10 $.3168 /$ jds.2018-16048) because their CR were $<0.01 \%$. These data revealed the effectiveness of using molecular descriptors of quinolones in assisted screening of heterologous coating antigens of interest.

The sensitivity of ELISA or other immunoassays sometimes did not meet the requirements set by authorities, after the preparation of $\mathrm{mAb}$, which involved lengthy and cumbersome processes. Many researchers have adopted quantitative structure-activity relationships (Wang et al., 2015; Chen et al., 2017a) to provide theoretical guidance for the design of novel haptens to produce high-performance mAb. However, using the prepared $\mathrm{mAb}$ with novel immunogens did not improve the sensitivity of immunoassays because of many uncertainties during $\mathrm{mAb}$ preparation, especially the different immune responses of model animals to immunogens. The heterologous competitive strategy has been confirmed to enhance the sensitivity of immunoas-

Table 3. Median inhibitory concentration $\left(\mathrm{IC}_{50}\right)$ values of ELISA with 25 coating antigens for the detection of enrofloxacin in PBS

\begin{tabular}{ll}
\hline Coating antigen $^{1}$ & $\mathrm{IC}_{50}{ }^{2}(\mathrm{ng} / \mathrm{mL})$ \\
\hline SAR-BSA & 0.13 \\
FLE-BSA & 0.21 \\
PEF-BSA & 0.26 \\
NOR-BSA & 0.36 \\
PRU-BSA & 0.49 \\
DAN-BSA & 1.28 \\
ENR-BSA & 1.32 \\
PIP-BSA & - \\
BAL-BSA & - \\
BES-BSA & - \\
CIN-BSA & - \\
CLI-BSA & - \\
GEM-BSA & - \\
LOM-BSA & - \\
MAR-BSA & - \\
MOX-BSA & - \\
NAL-BSA & - \\
ORB-BSA & - \\
OXO-BSA & - \\
PAZ-BSA & - \\
SIT-BSA & - \\
SPA-BSA & - \\
FLO-BSA & - \\
SMZ-BSA & - \\
TET-BSA & - \\
\hline
\end{tabular}

${ }^{1} \mathrm{SAR}=$ sarafloxacin $; \mathrm{FLE}=$ fleroxacin $; \mathrm{PEF}=$ pefloxacin $; \mathrm{NOR}=$ norfloxacin; PRU = prulifloxacin; DAN = danofloxacin; ENR = enrofloxacin; $\mathrm{PIP}=$ pipemidic acid; $\mathrm{BAL}=$ balofloxacin; $\mathrm{BES}=$ besifloxacin; $\mathrm{CIN}=$ cinoxacin; $\mathrm{CLI}=$ clinafloxacin; $\mathrm{GEM}=$ gemifloxacin; $\mathrm{LOM}$ $=$ lomefloxacin $; \mathrm{MAR}=$ marbofloxacin MOX $=$ moxifloxacin; $\mathrm{NAL}=$ nalidixic acid; ORB = orbifloxacin; $\mathrm{OXO}=$ oxolinic acid; $\mathrm{PAZ}=$ pazufloxacin; SIT = sitafloxacin; SPA = sparfloxacin; FLO = florfenicol; $\mathrm{SMZ}=$ sulfamethazine; $\mathrm{TET}=$ tetracycline.

${ }^{2} \mathrm{The} \mathrm{IC}_{50}$ values are invalid when the recognition ability for antigenantibody is weak. 
says (Goodrow et al., 1990; Zhang et al., 2007). Most researchers have selected suitable heterologous coating antigens to improve the sensitivity of immunoassays based on a large amount of experimental data. In our work, the molecular descriptors of haptens were used successfully in assisted screening of the qualified heterologous coating antigens to enhance the sensitivity of ELISA based on the machine learning method; this approach could save considerable time and money. The proposed strategy may provide new insight to improve the sensitivity of immunoassays.

However, the proposed strategy could only screen heterologous coating antigens of existing drugs. A future direction of our work is to screen the best heterologous coating antigens for a specific antibody in existing and novel drugs by using our pipeline. A key insight is that novel drugs will be designed based on the results of molecular docking between the complementaritydetermining region of antibody and epitopes of existing antigens (Wang et al., 2007, 2018).

\section{Analysis of Recognition Ability Between mAb and Heterologous Coating Antigen of Interest}

Interestingly, the $\mathrm{IC}_{50}$ of PIP-BSA was invalid (as shown in Table 3), whereas free PIP molecules could be recognized by $\mathrm{mAb}$ on the ELISA platform $(\mathrm{CR}=$ 1.89). Generally, Ag-Ab identification is influenced by many factors, such as electrostatic interaction, steric hindrance, van der Waals forces, and hydrogen bonding. A key factor that many researchers have focused on is the electrostatic interaction between $\mathrm{Ag}-\mathrm{Ab}$ (Wang et al., 2007). Molecular docking can reveal the electronic properties of targets, thereby influencing the identification of Ag-Ab (Ferreira et al., 2015). In this work, we established the electrostatic potential distribution (EPD) of epitopes by coupling the haptens with lysine to explain why mAb could not identify PIP-BSA on the ELISA system. The charge distribution of the epitope of ENR-BSA (epitope ENR-BSA) strongly matched that of the complementarity-determining region of $\mathrm{mAb}$ (strong electrostatic interaction) because the mAb was prepared with ENR-BSA as immunogen. As shown in Figure 2, more negative charges were found in block $\mathrm{A}^{*}$ (epitope PIP-BSA) than in block A (epitope ENR-BSA). Moreover, the negative and positive charges were denser in block $\mathrm{B}^{*}$ than in block $\mathrm{B}$, and the distribution of the positive and negative charges in block $\mathrm{B}^{*}$ was complicated compared with that in block B. Hence, the electrostatic interactions between the complementarity-determining region of $\mathrm{mAb}$ and epitope PIP-BSA were weak, making it difficult for PIP-BSA to be identified by mAb. The EPD of epitope DAN-BSA, epitope FLE-BSA, and epitope NOR-BSA were similar to that of epitope ${ }_{\text {ENR-BSA }}$; therefore, these 3 heterologous coating antigens could be recognized by mAb. In addition, compared with the EPD of epitope ENR-BSA, the EPD of epitope ${ }_{\text {SAR-BSA }}$, epitope PRU-BSA, and epitope NOR-BSA were slightly different, demonstrating that there were more negative than positive charges at the tip (away from lysine) of these epitopes. These differences adversely affected recognition between $\mathrm{mAb}$ and SAR-BSA, PRU-BSA, and NOR-BSA.

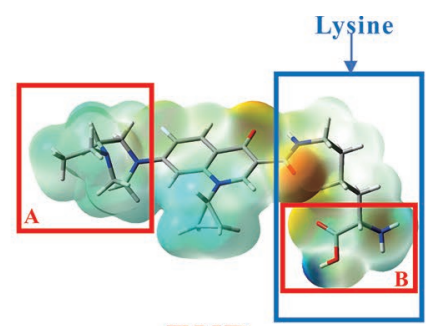

ENR

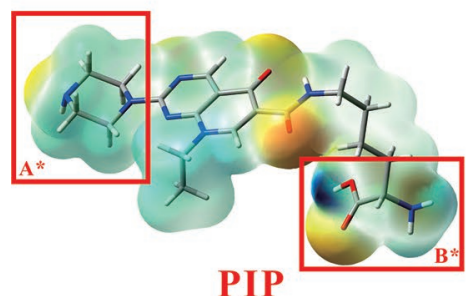

PIP

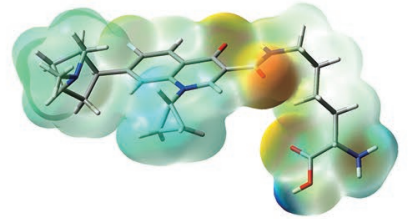

DAN

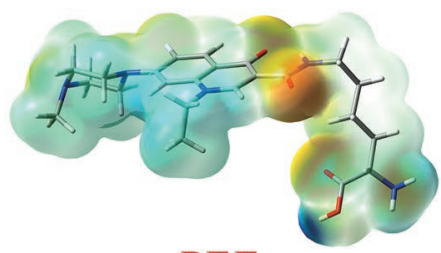

PEF
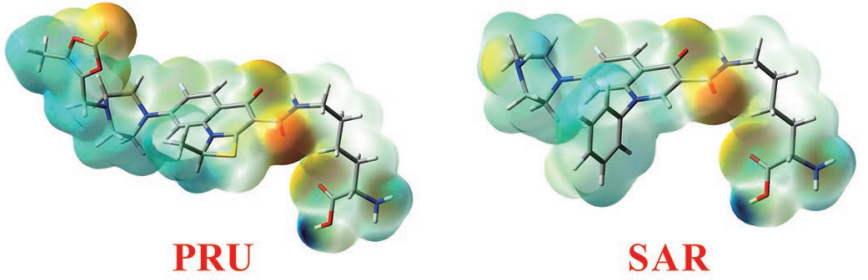

SAR

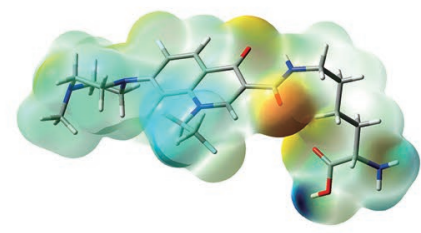

FLE

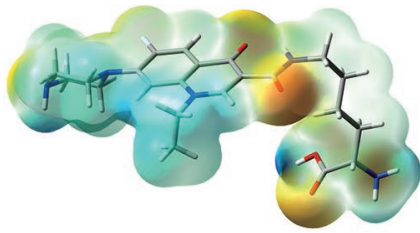

NOR

Figure 2. The electrostatic potential distribution of epitopes of 8 coating antigens. The negative charge is red, whereas the positive charge is blue. The structure in the blue box is lysine. The red boxes, A and B, represent the tip and end of the epitope to ENR-BSA, respectively; A* and $\mathrm{B}^{*}$ were the tip and end of the epitope to PIP-BSA, respectively. ENR = enrofloxacin; DAN = danofloxacin; PRU = prulifloxacin; SAR = sarafloxacin; PIP = pipemidic acid; PEF $=$ pefloxacin; FLE $=$ fleroxacin; NOR $=$ norfloxacin . 


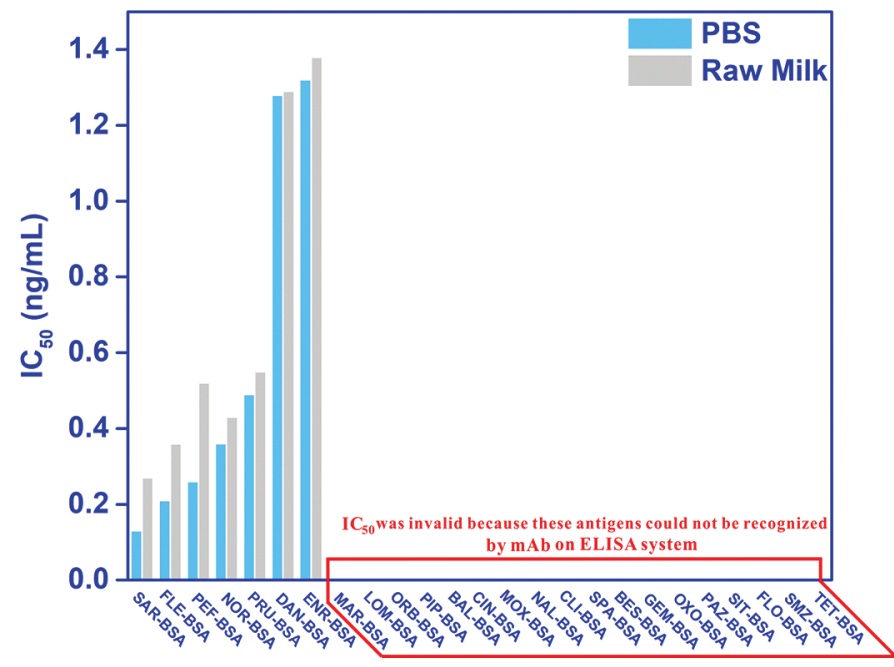

Figure 3. The sensitivity of ELISA for detection of ENR in raw milk and PBS. The sensitivity of ELISA was improved 6 -fold by using SAR-BSA as the coating antigen compared with ENR-BSA as the coating antigen. The sensitivity of ELISA in raw milk was less than that in PBS under the same coating antigen. SAR = sarafloxacin; FLE $=$ fleroxacin $; \mathrm{PEF}=$ pefloxacin; $\mathrm{NOR}=$ norfloxacin PRU $=$ prulifloxacin; $\mathrm{DAN}=$ danofloxacin; $\mathrm{ENR}=$ enrofloxacin; $\mathrm{MAR}=$ marbofloxacin; LOM = lomefloxacin; ORB = orbifloxacin; PIP = pipemidic acid; $\mathrm{BAL}=$ balofloxacin; $\mathrm{CIN}=$ cinoxacin; $\mathrm{MOX}=$ moxifloxacin $\mathrm{NAL}=$ nalidixic acid; CLI = clinafloxacin; $\mathrm{SPA}=$ sparfloxacin; $\mathrm{BES}$ = besifloxacin; GEM = gemifloxacin; $\mathrm{OXO}=$ oxolinic acid; $\mathrm{PAZ}=$ pazufloxacin; SIT = sitafloxacin; FLO = florfenicol; SMZ = sulfamethazine; $\mathrm{TET}=$ tetracycline $\mathrm{IC}_{50}=$ median inhibitory concentration.

\section{Sensitivity of ELISA for Detection of ENR in Raw Milk}

To further verify the advantage of the selected heterologous competitive antigens, we determined the sensitivity of ELISA for detecting ENR in several positive raw milk samples with ENR-BSA and suitable heterologous competitive antigens as coating antigens, respectively. As shown in Figure 3, the $\mathrm{IC}_{50}$ of ELISA for detection of ENR with 6 suitable heterologous competitive antigens as coating antigen were less than that with homologous antigen (ENR-BSA) as coating antigen, and the $\mathrm{IC}_{50}$ of ELISA was enhanced 6 -fold by SAR-BSA as coating antigen compared with ENR$\mathrm{BSA}$ as coating antigen. Additionally, the $\mathrm{IC}_{50}$ values of ELISA were invalid with other heterologous competitive antigens that could not be recognized by $\mathrm{mAb}$ on ELISA system as coating antigens. Notably, sensitivity of ELISA in raw milk was less than that in PBS under the same coating antigen integrally, which might be induced by matrix effect (Monaci et al., 2011; Brandon and Adams, 2015).

In the icELISA procedure, both the coating antigen and the small target molecule are capable of binding to the mAb. When the affinity between the coating antigen and $\mathrm{mAb}$ decreases, the sensitivity of ELISA is improved because the $\mathrm{mAb}$ is more likely to combine with the small molecule. In this work, the ELISA data (Supplemental Figure S6; https://doi.org/10.3168/jds .2018-16048) and the docking results (Figure 2) illustrated that the affinity of ideal heterologous competitive antigens with $\mathrm{mAb}$ was less than that of homologous antigen (ENR-BSA) with mAb. The ideal heterologous competitive antigens selected by molecular descriptors and ensemble learning improved the sensitivity of ELISA, as we expected.

\section{Recovery of ENR in Raw Milk on the ELISA System Using SAR-BSA as Coating Antigen}

The use of SAR-BSA as a coating antigen could maximally enhance ELISA sensitivity. The recovery of ENR in raw milk, therefore, was determined with SAR-BSA as coating antigen. The standard curve of the ELISA was constructed by plotting the OD value against the logarithm of the concentrations of ENR. The regression equation of the ELISA (Supplemental Figure S7; https://doi.org/10.3168/jds.2018-16048) was $y=-0.7383 \log (x)+0.3675\left(\mathrm{R}^{2}=0.9777\right)$. The recovery of ELISA for the determination of 0.1, 0.5, and $2 \mathrm{ng} / \mathrm{mL}$ ENR in raw milk was examined. Supplemental Table S4 (https://doi.org/10.3168/jds.2018-16048) shows average ELISA recoveries of 96.3 to $109.2 \%$ with CV of 1.8 to $6.7 \%$. Meanwhile, the recovery of ENR in raw milk with the ENR-BSA as coating antigen was determined (Supplemental Table S4; https://doi.org/ 10.3168/jds.2018-16048). These data indicated that the ELISA could reliably and precisely identify ENR in raw milk samples.

\section{Assay Validation}

Supplemental Table S5 (https://doi.org/10.3168/jds .2018-16048) showed detection results of 25 raw milk samples using the proposed ELISA and HPLC-MS/MS. Detailed information of HPLC-MS/MS data is shown in Supplemental File S1; https://doi.org/10.3168/jds .2018-16048). The data obtained by the 2 methods showed that 1 of the 25 raw milk samples was positive (sample 8). The detection result of the ELISA was compatible with that of the HPLC-MS/MS method.

\section{CONCLUSIONS}

In this work, we successfully prepared anti-ENR mAb and used the ensemble learning method to elucidate the relationship between molecular descriptors and CR classification (0 or 1$)$. The classification categories of the 7 testing samples were subsequently predicted with an accuracy of $100 \%$ by using their molecular descriptors. Seven heterologous coating antigens were 
prepared by conjugating BSA with the 7 heterologous quinolone drugs (category 1 in the training and testing samples). Six of the 7 heterologous coating antigens could enhance the sensitivity of ELISA; a key insight was that the sensitivity of ELISA with SAR-BSA as heterologous coating antigen was enhanced 10-fold in PBS and 6-fold in raw milk compared with that when ENR-BSA was used as coating antigen. Our pipeline can effectively screen for suitable heterologous coating antigens of interest to improve the sensitivity of ELISA.

\section{ACKNOWLEDGMENTS}

This work was supported by the National Science Foundation for Young Scientists of China (Beijing, China; 31800776), Jiangxi Science Foundation for Young Scientists (Nanchang, China; 20181BAB214018), Jiangxi Special Fund for Graduate Innovation Research (Nanchang, China; YC2018-S007), Jiangxi Special Fund for Agro-scientific Research in the Collaborative Innovation (Nanchang, China; JXXTCX201703-1), and earmarked fund for Jiangxi Agriculture Research System (Nanchang, China; JXARS-03). The authors declare no conflicts of interest.

\section{REFERENCES}

Ballabio, D. 2015. A MATLAB toolbox for principal component analysis and unsupervised exploration of data structure. Chemom. Intell. Lab. Syst. 149:1-9.

Barberio, A., B. Flaminio, S. D. Vliegher, K. Supré, V. Kromker, C. Garbarino, N. Arrigoni, G. Zanardi, L. Bertocchi, and F. Gobbo. 2016. Short communication: In vitro antimicrobial susceptibility of Mycoplasma bovis isolates identified in milk from dairy cattle in Belgium, Germany, and Italy. J. Dairy Sci. 99:6578-6584.

Bianchi, F., M. Careri, M. Musci, and A. Mangia. 2007. Fish and food safety: Determination of formaldehyde in 12 fish species by SPME extraction and GC-MS analysis. Food Chem. 100:1049-1053.

Bober, L., M. Koba, U. Judycka-Proma, and T. Baczek. 2011. Pharmacological classification of drugs by principal component analysis applying molecular modeling descriptors and HPLC retention data. J. Chromatogr. Sci. 49:758-763.

Brandon, D. L., and L. M. Adams. 2015. Milk matrix effects on antibody binding analyzed by enzyme-linked immunosorbent assay and biolayer interferometry. J. Agric. Food Chem. 63:3593-3598.

Chen, J., L. Wang, L. Lu, X. Shen, X. Huang, Y. Liu, X. Sun, Z. Wang, S. A. Eremin, and Y. Sun. 2017a. Four specific hapten conformations dominating antibody specificity: Quantitative structure-activity relationship analysis for quinolone immunoassay. Anal. Chem. 89:6740-6748.

Chen, Y., L. Guo, L. Liu, S. Song, H. Kuang, and C. Xu. 2017b. Ultrasensitive immunochromatographic strip for fast screening of 27 sulfonamides in honey and pork liver samples based on a monoclonal antibody. J. Agric. Food Chem. 65:8248-8255.

Colgrave, M. L., H. Goswami, M. Blundell, C. A. Howitt, and G. J. Tanner. 2014. Using mass spectrometry to detect hydrolysed gluten in beer that is responsible for false negatives by ELISA. J. Chromatogr. A 1370:105-114.

EMEA (European Medicines Agency). 2002. Enrofloxacin (extension to all food producing species) - Summary report. EMEA/ MRL/820/02-FINAL. Committee for Veterinary Medical Products/European Agency for the Evaluation of Medicinal Products. EMEA, Amsterdam, the Netherlands.
Ershadi, S., A. Jouyban, and A. Shayanfar. 2017. Determination of enrofloxacin in milk samples using silver nanoparticle enhanced terbium-sensitized fluorescence method. Food Anal. Methods 10:1-8.

Ferreira, L. G., R. Dos Santos, G. Oliva, and A. Andricopulo. 2015. Molecular docking and structure-based drug design strategies. Molecules 20:13384-13421.

Goodrow, M. H., R. O. Harrison, and B. D. Hammock. 1990. Hapten synthesis, antibody development, and competitive inhibition enzyme immunoassay for s-triazine herbicides. J. Agric. Food Chem. 38:990-996.

Guidi, L. R., F. A. Santos, R. Acsr, C. Fernandes, S. Lhm, and G. Mba. 2018. Quinolones and tetracyclines in aquaculture fish by a simple and rapid LC-MS/MS method. Food Chem. 245:1232-1238.

Hosoda, H., N. Kawamura, and T. Nambara. 1981. Effect of bridge heterologous combination on sensitivity in enzyme immunoassay for cortisol. Chem. Pharm. Bull. (Tokyo) 29:1969-1974.

Hu, L. M., K. Luo, J. Xia, G. M. Xu, C. H. Wu, J. J. Han, G. G. Zhang, M. Liu, and W. H. Lai. 2017. Advantages of time-resolved fluorescent nanobeads compared with fluorescent submicrospheres, quantum dots, and colloidal gold as label in lateral flow assays for detection of ractopamine. Biosens. Bioelectron. 91:95-103.

Huang, X., Y. Liu, B. Yung, Y. Xiong, and X. Chen. 2017. Nanotechnology-enhanced no-wash biosensors for in vitro diagnostics of cancer. ACS Nano 11:5238-5292.

Luo, Y., X. Zhao, J. Zhou, J. Yang, Y. Zhang, W. Kuang, J. Peng, L. Chen, and J. Zeng. 2017. A network integration approach for drugtarget interaction prediction and computational drug repositioning from heterogeneous information. Nat. Commun. https://doi.org/ 10.1038/s41467-017-00680-8.

Monaci, L., M. Brohée, V. Tregoat, and A. V. Hengel. 2011. Influence of baking time and matrix effects on the detection of milk allergens in cookie model food system by ELISA. Food Chem. 127:669-675.

Otero, J. A., D. García-Mateos, A. D. L. Fuente, J. G. Prieto, A. I. Álvarez, and G. Merino. 2016. Effect of bovine ABCG2 Y581S polymorphism on concentrations in milk of enrofloxacin and its active metabolite ciprofloxacin. J. Dairy Sci. 99:5731-5738.

Peng, J., D. Kong, L. Liu, S. Song, H. Kuang, and C. Xu. 2015. Determination of quinoxaline antibiotics in fish feed by enzyme-linked immunosorbent assay using a monoclonal antibody. Anal. Methods 7:5204-5209.

Peng, J., L. Liu, H. Kuang, G. Cui, and C. Xu. 2016. Development of an icELISA and immunochromatographic strip for detection of norfloxacin and its analogs in milk. Food Agric. Immunol. 28:288298

Peng, J., L. Liu, L. Xu, S. Song, H. Kuang, G. Cui, and C. Xu. 2017. Gold nanoparticle-based paper sensor for ultrasensitive and multiple detection of 32 (fluoro)quinolones by one monoclonal antibody. Nano Res. 10:108-120.

Pereira, R. V., J. D. Siler, J. C. Ng, M. A. Davis, Y. T. Grohn, and L. D. Warnick. 2014. Effect of on-farm use of antimicrobial drugs on resistance in fecal Escherichia coli of preweaned dairy calves. J. Dairy Sci. 97:7644-7654.

Perosa, F., R. Carbone, S. Ferrone, and F. Dammacco. 1990. Purification of human immunoglobulins by sequential precipitation with caprylic acid and ammonium sulphate. J. Immunol. Methods 128:9-16.

Rao, P. N., and I. B. Taraporewala. 1992. A sensitive enzyme-linked immunosorbent assay (ELISA) for testosterone: use of a novel heterologous hapten conjugated to penicillinase. Steroids 57:154-161.

Rassaie, M. J., G. L. Kumari, P. N. Rao, T. G. Shrivastav, and H. P. Pandey. 1992. Influence of different combinations of antibodies and penicillinase-labeled testosterone derivatives on sensitivity and specificity of immunoassays. Steroids 57:112-118.

Rosen, G. L., E. R. Reichenberger, and A. M. Rosenfeld. 2011. NBC: The naive Bayes classification tool webserver for taxonomic classification of metagenomic reads. Bioinformatics 27:127-129.

Shen, H., and K. C. Chou. 2005. Using optimized evidence-theoretic $\mathrm{K}$-nearest neighbor classifier and pseudo-amino acid composition to predict membrane protein types. Biochem. Biophys. Res. Commun. 334:288-292. 
Stuhlsatz, A., J. Lippel, and T. Zielke. 2012. Feature extraction with deep neural networks by a generalized discriminant analysis. IEEE Trans. Neural Netw. Learn. Syst. 23:596-608.

Tang, Y., Y. Zhang, H. Zhang, X. Liu, X. Gao, C. Lv, T. Ma, X. Lu, and J. Li. 2017. Visual flow-through column biomimetic immunoassay using molecularly imprinted polymer as artificial antibody for rapid detection of clenbuterol in water sample. Food Agric. Immunol. 28:1-9.

Tochi, B. N., G. Khaemba, J. Isanga, D. Mukunzi, L. Liu, J. Peng, H. Kuang, and C. Xu. 2015. Monoclonal antibody for the development of specific immunoassays to detect enrofloxacin in foods of animal origin. Food Agric. Immunol. 27:435-448.

Turiel, E., A. Martín-Esteban, and J. L. Tadeo. 2006. Multiresidue analysis of quinolones and fluoroquinolones in soil by ultrasonicassisted extraction in small columns and HPLC-UV. Anal. Chim. Acta 562:30-35.

Ukil, A. 2002. Support vector machine. Comput. Sci. 1:1-28.

Wang, L., W. M. Jiang, X. Shen, X. Li, X. Huang, Z. Xu, Y. Sun, S. W. Chan, L. Zeng, and S. A. Eremin. 2018. Four hapten spacer sites modulating class specificity: non-directional multianalyte immunoassay for $31 \beta$-agonists and analogues. Anal. Chem. 90:2716-2724.

Wang, Z., M. Liu, W. Shi, C. Li, S. Zhang, and J. Shen. 2015. New haptens and antibodies for ractopamine. Food Chem. 183:111-114.

Wang, Z., H. Zhang, H. Ni, S. Zhang, and J. Shen. 2014. Development of a highly sensitive and specific immunoassay for enroflox- acin based on heterologous coating haptens. Anal. Chim. Acta 820:152-158.

Wang, Z., Y. Zhu, S. Ding, F. He, R. C. Beier, J. Li, H. Jiang, C. Feng, Y. Wan, and S. Zhang. 2007. Development of a monoclonal antibody-based broad-specificity ELISA for fluoroquinolone antibiotics in foods and molecular modeling studies of cross-reactive compounds. Anal. Chem. 79:4471-4483.

Yang, Y., Y. Wang, N. Rui, J. Lu, X. Zhu, and W. Yun. 2016. Preparation and characterization of chitosan microparticles for immunoaffinity extraction and determination of enrofloxacin. Int. J. Biol. Macromol. 93(Pt A):783-788.

Yu, X., X. Zhang, Z. Wang, H. Jiang, Z. Lv, J. Shen, G. Xia, and K. Wen. 2018. Universal simultaneous multiplex ELISA of smal molecules in milk based on dual luciferases. Anal. Chim. Acta 1001:125-133.

Zhang, Y., S. Lu, W. Liu, C. Zhao, and R. Xi. 2007. Preparation of anti-tetracycline antibodies and development of an indirect heterologous competitive enzyme-linked immunosorbent assay to detect residues of tetracycline in milk. J. Agric. Food Chem. 55:211-218.

Zhao, Y., G. Zhang, Q. Liu, M. Teng, J. Yang, and J. Wang. 2008 Development of a lateral flow colloidal gold immunoassay strip for the rapid detection of enrofloxacin residues. J. Agric. Food Chem. 56:12138-12142. 